\title{
Introducing an Image Processing Method for Evaluation of Clean and Jerk Style in Weightlifting using Measured Biomechanical Parameters
}

\author{
Mohammad Almasi \\ Departments of Biometrics, University of Paris Est-Creteil
}

\begin{abstract}
Weightlifting is one of the high-medal sports in the Olympic Games. Although one of the most critical weaknesses of this sport is the lack of using new method of technology to improve the quality of player's performance and also decline the referee mistakes. The aim of this paper is to use Image processing to figure out the clinical parameters to examine and measuring the angular kinematic and kinetic features of clean and jerk style in weightlifting. And using this approach for defining the standard pattern for distinguishing the difference between correct and wrong movements that can be used in talent identification.
\end{abstract}

\section{Keywords}

Weightlifting, Clean and Jerk style, Image processing, Computer Vision, Olympics Games.

\section{INTRODUCTION}

Weightlifting with 16 gold medal probability has one of the first medal ranking among the other Olympic Games sports [1]. One of the problems with this sport is the lack of engineering analysis and the lack of technology usage for improving weightlifters' quality in terms of kinematic and kinetic parameters of their movements which can enhance their performance level. The use of technology has always been one of the most widely used methods for simplifying complex problems in nature. Sports is no exception and there is hope to improve athlete's quality using engineering techniques. Sports engineering is one of the fields of engineering science which aims to facilitate sports processes such as athlete's performance, sports equipment's, stadiums and other dominating factors $[2,3]$. One of the methods not only for analyzing athlete's performance but also for talent identification is using the image processing. Furthermore this method has applications in referee systems in various sports [4]. For instance, in Volleyball, the video challenge system can be used to judge the event with a higher precision [5] .or in swimming, it can be used to obtain information about an athlete's performance in order to improve it [6]. Or in table tennis as one the most popular sports among young generations sports performance analysis can evaluate and improve their level as done by [7]. So, in weightlifting, the image processing systems can be used not only to reduce the referee's mistakes but also to improve an athlete's performance in terms of kinematic and kinetic dimensions to perform the movements correctly. This paper tries to investigate the correct performance of each movement in relation to a certain body part and parameter through image processing using ordinary cameras after identification of significant biomechanical parameters in the athlete's body including angle, angular velocity and angular acceleration in various parts of body such as wrist, elbow, shoulder and knee. Then, similar procedure was performed for cameras with high frame rate. Finally, a comparison was made between these two types of camera. The cameras with high frame rate have various applications in sports. For example the cameras with high frame rate are used in soccer as goal line technology [8]. Though, present research examines to indicate that the camera type does not make any significant changes in the research results of weightlifting. However, the high frame rate cameras indicate a higher precision. In last but not least, a method for discriminating between correct and incorrect movements was mentioned.

\section{METHODOLOGY}

In this research, a set of experiments were carried out on the four joints include of wrist, elbow, shoulder and knee due to these parts are more sensitive than other part in jury decision or have a correct lift without injury [9]. Five professional weightlifters were helped in this issue, as shown in table 1 their anthropometric characters and the speed and their record weight is comprised $[10,11]$.

Table1. Anthropometric Features of Five players that participated in test

\begin{tabular}{|l|l|l|l|}
\hline Features & HEIGHT & WEIGHT & SPEED \\
\hline 1 & & & \\
\hline 2 & 197 & 85 & 4.1 \\
\hline 3 & 175 & 85 & 5.5 \\
\hline 4 & 176 & 85 & 5.0 \\
\hline 5 & 181 & 85 & 4.0 \\
\hline
\end{tabular}

The image processing toolbox in MATLAB was used to find out the above-mentioned parameters for each joint during three pre-processing, processing and post-processing stages [12]. Now it should be noticed why angular parameters have been used. There are two main reasons for using angular parameters rather than linear parameters. First, a precise velocity and acceleration is only obtained through creating a correct angle among various joints of arm and leg. And second, the linear velocity and acceleration parameters, in anthropometrical model, cannot discriminate among individuals due to different length of each individual's arm or leg, the angular velocity and acceleration can predict results with higher precision [13]. One of the aims of this study was to analyze and discriminate between correct and incorrect movements. To achieve this goal, the incorrect movement should be defined. The incorrect movement refers to a movement that is correct from a referee's perspective but can cause injuries for athlete [14]. Estimating and analyzing movements in each joint in terms of the intended parameters after performing the clean and jerk style incorrectly. 
Therefore, this method can be used to compare and train the correct performance of this movement among athletes.

\section{RESULTS AND DISCUSSION}

In this study, first an ordinary camera with a 30 frames per second (fps) was used to understand the angular parameters from critical body features in clean and jerk style of weightlifting [15]. Then, a camera with a high frame rate was used. However, the high frame cameras are not only have high cost but also, these cameras can store and handle a high amount of data. That can reduce the processing speed, they occupy the central processing unit. However, there was no considerable distinction between these two cameras types for using in weightlifting in fixed position. Due to that, critical points are similar in these two camera types.

For defining the difference between low frame rate and high frame rate cameras it has be need to understand the most important and critical sequences, that mean those times with change in angular parameters for each joint. In clean and jerk style of two Scissoring and pushing up is more important than other times. Because the player need more energy and accelerate for them it can make some injuries [13]. As can be seen below in image one and two for shoulder the critical changed point between LFR and HFR are the same.

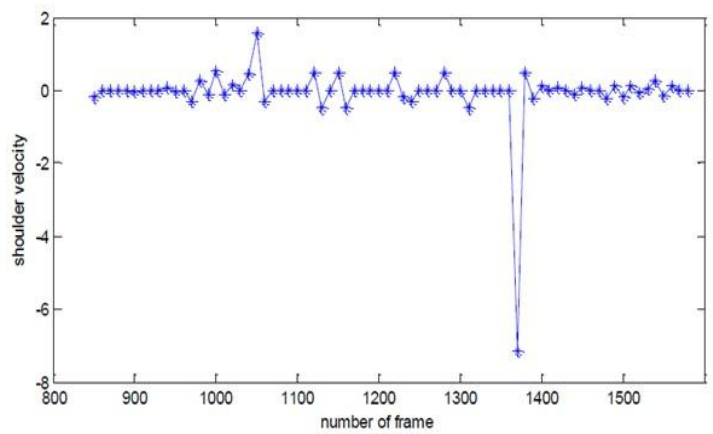

Fig1: High frame rate shoulder velocity

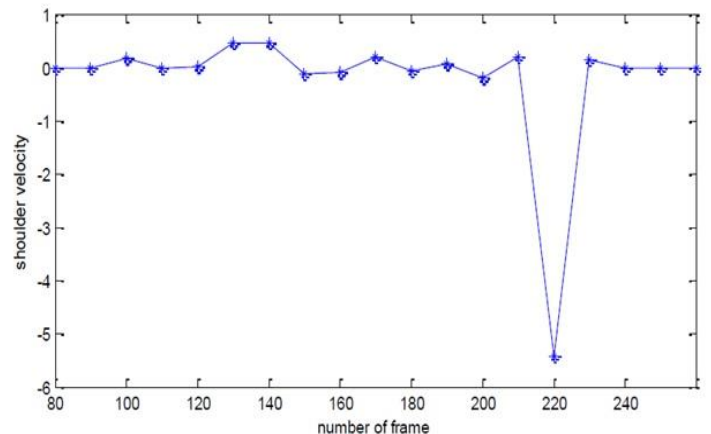

Fig2: Low frame rate shoulder velocity.

In above images the most important sequence is when the player want to lift the weight up to his head, and push it up. So he should use more energy to lift it up. For analyzing the elbow joint it can be demonstrated as below, that in two times the most changes was happening. That the first one is for lifting from the surface and the second is for pushing the weight up to his head.

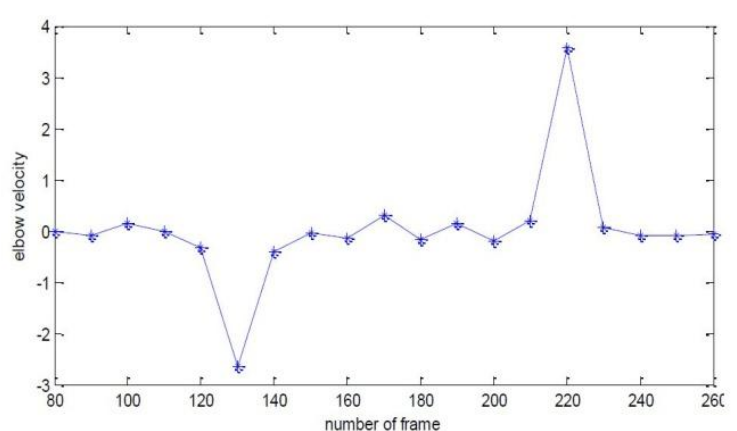

Fig3: Low frame rate elbow velocity.

As can be seen in below, the most critical time changing is the same, in LFR and HFR but it had has shown some unnecessary information that can be ignored.

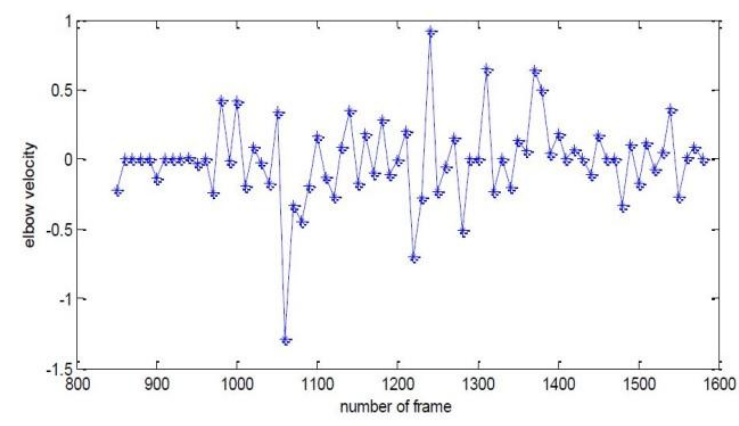

Fig4: High frame rate elbow velocity.

It can be shown for other joints too. But as can be shown above, all critical points were the same in high frame rate and low frame rate cameras, in considering the sequences of change, so it could be possible to use ordinary cameras. In order to understand and comparison between correct and incorrect movements, not only there should have been a pattern for comparing. Also considering to the critical time of changes is important, furthermore remember, the definition of incorrect movement is that make injury rather than negative point from referee. As shown below in incorrect results for angle it can be seen that the player start to change the position of elbow a bit earlier than the correct pattern, while in correct pattern the 135 is the frame for start elbow change and in wrong one he started from 23 second and it means he started to lift up the weight to head too early. And this incorrect procedure will give other results in velocity or acceleration incorrect.

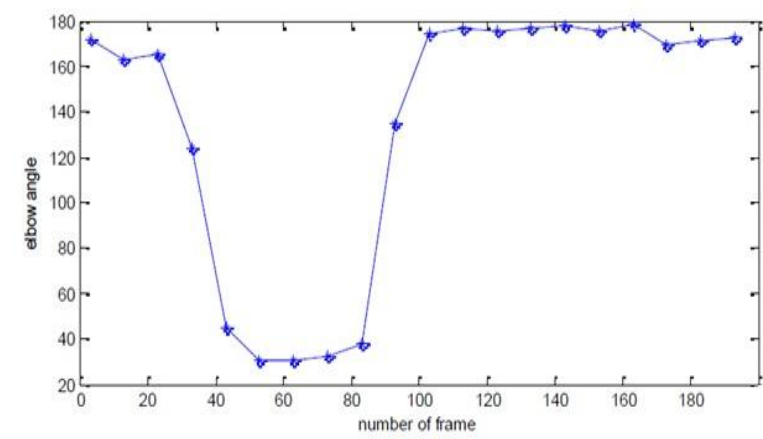

Fig5: Incorrect angle of elbow. 


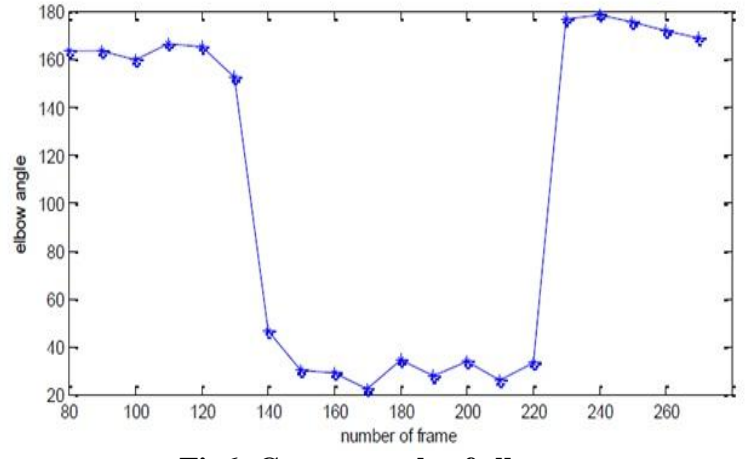

Fig6: Correct angle of elbow.

For another example in distinguishing between correct and incorrect pattern, in below, it can be seen for shoulder angle that, due to fixing and maintenance the elbow angle he should change the whole body position.

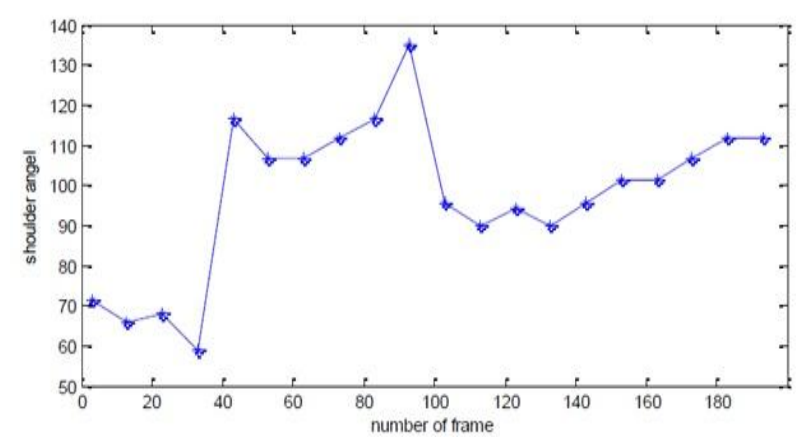

Fig7: Incorrect angle of shoulder.

But in correct pattern as shown below, it does not necessary to change the whole body for maintenance the movements. Until the player want to do the scissoring part.

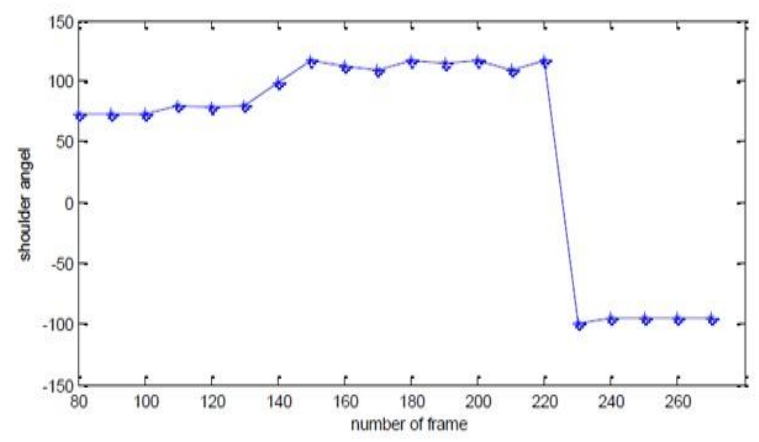

Fig8: Correct angle of shoulder.

In term of understanding the level of incorrect movement, the shoulder acceleration will examined, and due to below figures, it can be seen that amateur player has had considerable problems in lifting and scissoring. And it can be seen more accurately in acceleration results. As shown below in shoulder part he cannot manage the change times, while in figure 10, the correct change to lift and open the shoulders is at the end of the time, by a professional player.

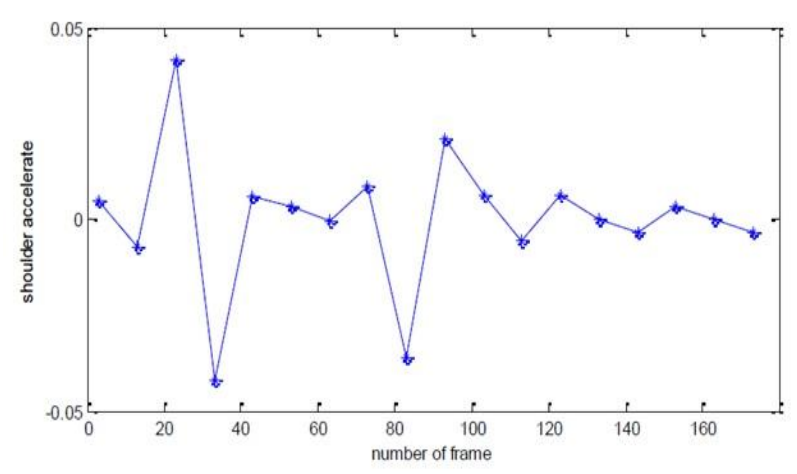

Fig9: Incorrect acceleration of shoulder.

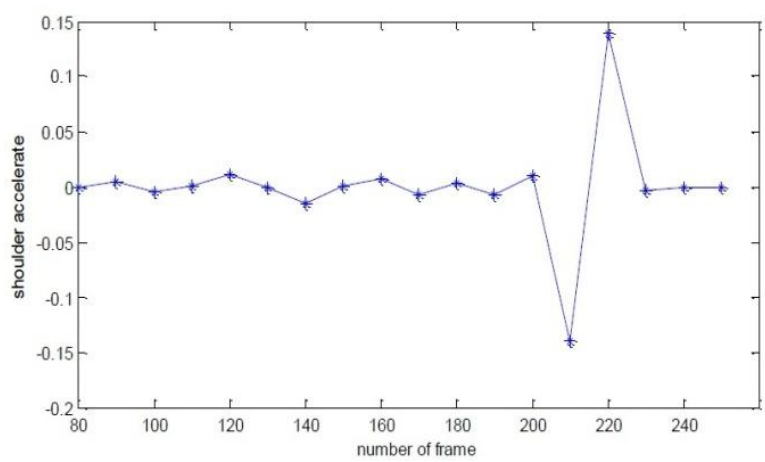

Fig10: Correct acceleration of shoulder.

\section{CONCLUSION}

This paper tries to propose a method for evaluating the correct performance of clean and jerk style in weightlifting using image processing techniques. Then, the results were analyzed using high frame rate cameras to make sure and see the different of using these two kind of cameras in weightlifting and to show, that except the general deference between high frame rate and low frame rate cameras the results was the same in critical and important points. So it could be possible to use ordinary cameras in weightlifting. Finally, the research results can be used to evaluate and enhance athlete performance level whether in high level athletes or among amateurs for talent identification using making comparison with Correct and Worng movemnts.

\section{REFERENCES}

[1]. "IWF Technical and Competition Rules" (PDF). International Weightlifting Federation. Retrieved 201701-10.

[2]. Peter E.Jenkins, Atousa Plaseied, Morteza khodae. (2010). UCD Sports Engineering Program. Procedia Engineering 2. 2757-2762.

[3]. Mohamad Almasi. Sports engineering program in Islamic Azad University branch of Research and science. UCT Journal of Research in Science, Engineering and Technology

(Volume4Issue2June2016).http://uctjournals.com/archive /ujrset/2016/June/7.pdf.

[4]. Girish G. Koundinya, G. Jaikumar, N. Rahul Akash and M.S.V. Subramanian. (2012). Survey on Digital Image Processing in Sports. Research Journal of Applied Sciences, Engineering and Technology 4(24): 5552 5556. ISSN: 2040-7467. 
[5]. Challenge System Regulations for Volleyball. International Federation of Volleyball (FIVB). December 2017,http://ebook.cev.lu/development/Referee/CEV_Cha llenge_System_Regulations_Volleyball.pdf.

[6]. Robin P. Dubois, David V.Thiel, Daniel A.James, .Using image processing for biomechanics measures in swimming. Procedia Engineering Volume 34, 2012, Pages 807-812. https://doi.org/10.1016/j.proeng.2012.04.138.

[7]. Mohamad Almasi. New method based on Image processing for improvement the Accuracy and Precision in table tennis player's performance. . UCT Journal of Research in Science, Engineering and Technology (Volume 4 Issue 3 Sep2016).http://uctjournals.com/archive/ujrset/2016/Sep/ 1.pdf

[8]. Rafael Psiuk, Thomas Seidl, Wolfram Strauß, Josef Bernhard. (2014). Analysis of Goal Lin Technology from the perspective of an electromagnetic field based approach. The 2014 Conference of international Sports engineering association. Procedia engineering. 72. 279284

[9]. Arthur E. Chapman. 2008. Biomechanical Analysis of Fundamental Human Movements. ISBN-13: 9780736064026 .
[10]. M ramezani, $\mathrm{F}$ yaghmaee, A novel Video recommendation system based on efficient retrieval of human actions,PhysicaA.(2016), http://dx.doi.org/10/2016/j.physa2106.03.101.

[11]. Xiaojing Zhou, Jinxiang Chen, Guohua Chen, Zhengxu Zhao, Yong Zhao. Anthropometric body modeling based on orthogonal-view images. International Journal of Industrial Ergonomics Volume 53, May 2016, Pages 2736. https://doi.org/10.1016/j.ergon.2015.10.007.

[12]. B. Chitradevi, P. Srimathi. (2014). An Overview on Image Processing Techniques. International Journal of Innovative Research in Computer and Communication Engineering. Vol. 2, Issue 11, November 2014.

[13]. marius viorel ulăreanu $v p$, olivia carmen timnea, cosmina cheran. (2014) Biomechanical characteristics of movement phases of clean \& jerk style in weightlifting performance. J Social and Behavioral Sciences137:6469. doi:10.1016/j.sbspro.2014.05.253.

[14]. Gregg Calhoon, Andrew C. Fry. (1999). Injury rates and profiles of elite competitive weightlifters. Journal of Athletic Training. 34(3):232-238.

[15]. Mohammad Almasi. 2017. Image processing application for measuring the Biomechanical parameters in Clean and Jerk style of weightlifting. 3rd.International Conference on Researches in Science \& Engineering. 